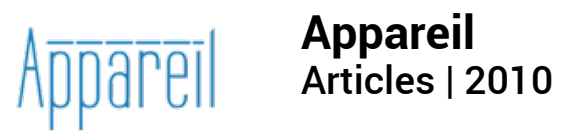

\title{
Fenêtres et écrans : entre intimité et extimité
}

Intervention aux journées d'étude des 2, 3, 4 juin 2009, à Tonnerre (Yonne), Arts et techniques, sous la responsabilité de J. L. Déotte avec l'appui de la revue en ligne Appareil, MSH Paris Nord, École doctorale "Pratiques et théories du sens ».

\section{Georges Teyssot}

\section{(2) OpenEdition Journals}

\section{Édition électronique}

URL : http://journals.openedition.org/appareil/1005

DOI : 10.4000/appareil.1005

ISSN : 2101-0714

Éditeur

MSH Paris Nord

\section{Référence électronique}

Georges Teyssot, «Fenêtres et écrans : entre intimité et extimité », Appareil [En ligne], Articles, mis en ligne le 09 mars 2010, consulté le 30 juillet 2020. URL : http://journals.openedition.org/appareil/1005 ; DOI : https://doi.org/10.4000/appareil.1005

Ce document a été généré automatiquement le 30 juillet 2020.

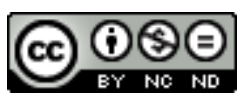

Appareil est mis à disposition selon les termes de la Licence Creative Commons Attribution - Pas d'Utilisation Commerciale - Pas de Modification 4.0 International. 


\section{Fenêtres et écrans : entre intimité et extimité}

Intervention aux journées d'étude des 2, 3, 4 juin 2009, à Tonnerre (Yonne), Arts et techniques, sous la responsabilité de J. L. Déotte avec l'appui de la revue en ligne Appareil, MSH Paris Nord, École doctorale «Pratiques et théories du sens ».

\section{Georges Teyssot}

\section{Traduit de l'anglais par Marion Delage de Luget, mariondelagedeluget@yahoo.fr}

Dès lors que l'on s'accorde à reconnaître la disparition actuelle des espaces publics traditionnels, redessiner la frontière entre les sphères publiques et privées s'impose comme une tâche impérieuse. La question qui se pose aujourd'hui est de comprendre si l'on peut imaginer un espace public fondé dans la pluralité, c'est-à-dire un espace public qui ne soit pas conçu comme un endroit unique, mais dans le cadre d'une multiplicité. Ce qui devrait peut-être alors être exploré, ce sont ces limites changeantes entre l'intimité et la publicité que les nouveaux médias ont ouvertes, permettant l'apparition d'un flâneur numérique et menant à une dérive mondiale. Ces nouvelles limites marquent des zones originales où l'on pourrait demeurer, singulièrement mais également collectivement. Actuellement, les problèmes qu'il est urgent de questionner concernent les sujets du «regard», les anxiétés soulevées par l'effacement de tout domaine privé, les préoccupations relatives à l'exhibition de l'intimité dans les médias, enfin la mutation de la notion de « milieu ». De plus, on pourrait se demander comment les notions de fenêtre, de porte, de cadre et d'écran ont évolué dans le temps; et comment, sous de nouveaux aspects, elles contribuent à l'apparition d'un terrain virtuel, ou de topographies numériques.

3 Les êtres humains pourraient être caractérisés comme des " animaux-à-fenêtres ", attendu qu'entre le monde et le sujet il $\mathrm{y}$ a toujours quelque chose qui opère une médiation; pas tellement, en soi, un médium, mais quelque chose qui connecte toutes ces choses extérieures, et nous. Dans son livre phare sur la Fenêtre (2004), le psychanalyste Gérard Wacjman rappelle qu'une «fenêtre » est non seulement un trope littéraire ou une métaphore, mais qu'elle est néanmoins, aussi, un véritable mécanisme, 
se référant par là à la fenêtre réelle. Une fenêtre littérale, construite avec un jeu de cadres et de carreaux de verre, protégée de l'extérieur par des volets et à l'intérieur par des rideaux. Cependant, si on pouvait arracher les montants fixes et les meneaux tenant les carreaux de verre, etc., il ne resterait qu'une simple ouverture, une baie ou un trou dans le mur qui laisse un regard vers l'extérieur, devenant ainsi un œil, un organe de vision. Et si la fenêtre peut être un œil, il en découle que l'œil peut être une fenêtre. Dans l'histoire humaine, beaucoup de choses ont été nos fenêtres : fenêtres construites, œil, lunettes, peintures, appareils photographiques, vidéos. Tous ces instruments nous aident à nous relier au monde dans lequel nous sommes constamment immergés.

4 Comme n'importe quel autre instrument (y compris le langage), la fenêtre est un appareil qui sépare et unit. Pour être capable de comprendre le monde, l'homme a besoin d'être équipé, parce qu'il est un être handicapé qui requiert beaucoup d'accessoires. ${ }^{1}$ C'est un fait connu que le mot anglais « window ", la fenêtre, provient du "vent» et de «l'œil», et cette étymologie aide à définir l'ouverture comme un instrument complexe, qui en premier lieu autorise la lumière et la ventilation, et devient ensuite un instrument permettant l'œil, c'est-à-dire la vision. ${ }^{2}$ En commençant avec le fameux traité de Leon Battista Alberti sur la peinture, la métaphore de la peinture est "une fenêtre ouverte [aperta finestra] au travers de laquelle on voit le sujet [historia] à peindre." Naturellement, une telle comparaison institue cette façon occidentale de voir le monde comme une image; elle transforme également la fenêtre réelle, qui, à l'époque d'Alberti, n'était ni carrée, ni parfaitement vitrée ou transparente.

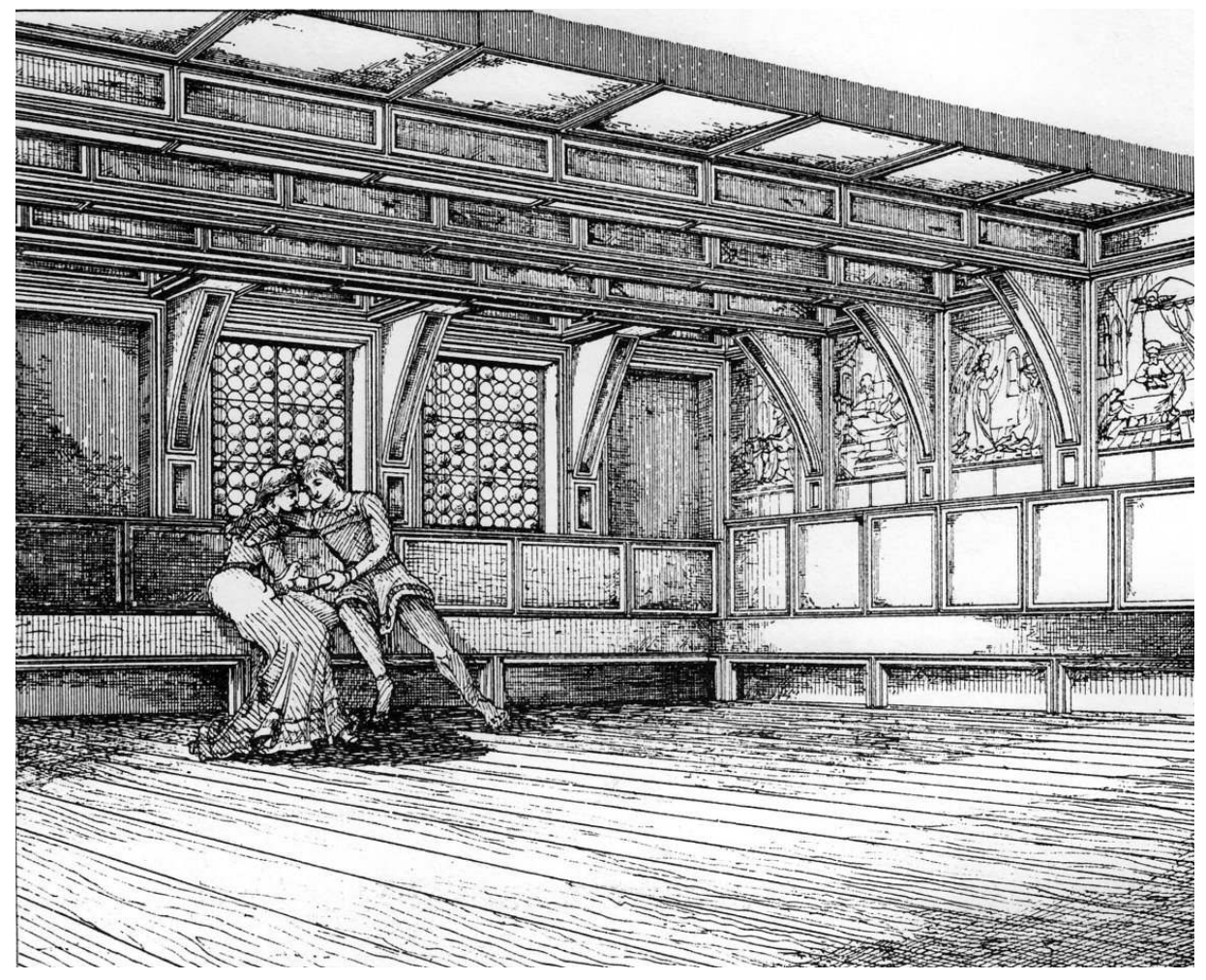

$5 \quad$ Fig. 1 - Fenêtre dans une résidence d'époque Renaissance, Moenchgasse, Goslar, Basse Saxe, Allemagne, 1562, détail, de : Karl Schaefer, Die Holzarchitektur Deutschlands vom XIV. bis XVIII. Jahrhundert, Verbande Deutscher Architekten - und Ingenieur - Vereine 
und dem Gesammtvereine der Deutschen Geschichts - und Alterthums - Vereine, Berlin: Wasmuth, 1883-1888, pl. 21.

6 À partir de la Renaissance, la fenêtre présente, d'un côté, son utilité comme moyen d'éclairage, de ventilation, d'isolation, etc., et, d'un autre côté, on la pense comme une chose au travers de laquelle on peut regarder. La fenêtre est un agencement « architectural » qui sert de dispositif utile et hygiénique, relié aux besoins du corps; et également un appareil «optique »- une sorte de loupe, ou comme des lunettes -, qui offre aussi une ouverture sur le monde.

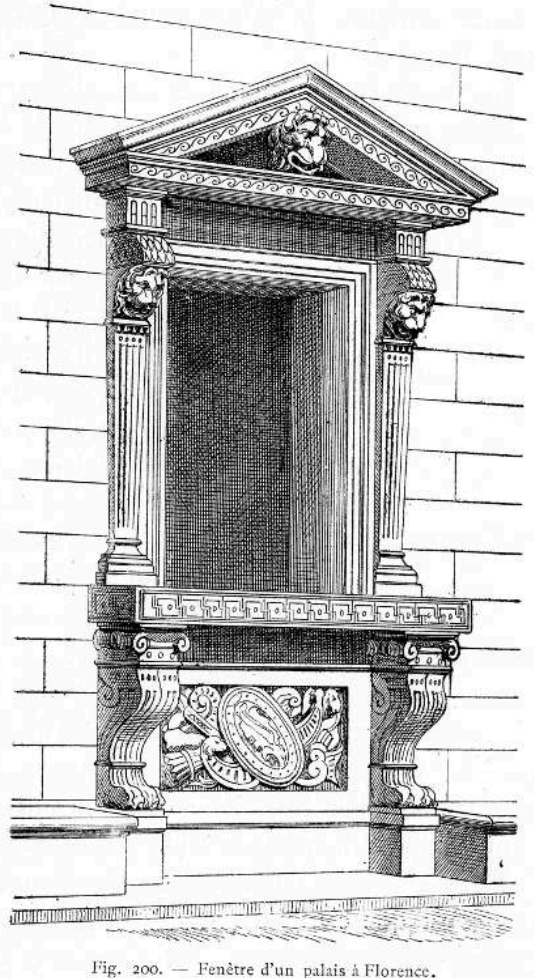

7 Fig. 2 - Fenêtre d'un palais à Florence, de : Julien Guadet, Éléments et théorie de

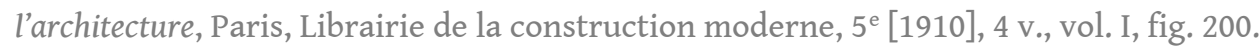

Dans ce dernier cas, tout se produit comme si la fenêtre avait été équipée d'un « œil artificiel ", comme cela apparaît dans les gravures illustrant l'Ars magna lucis et umbrae d'Athanasius Kircher (1646). 


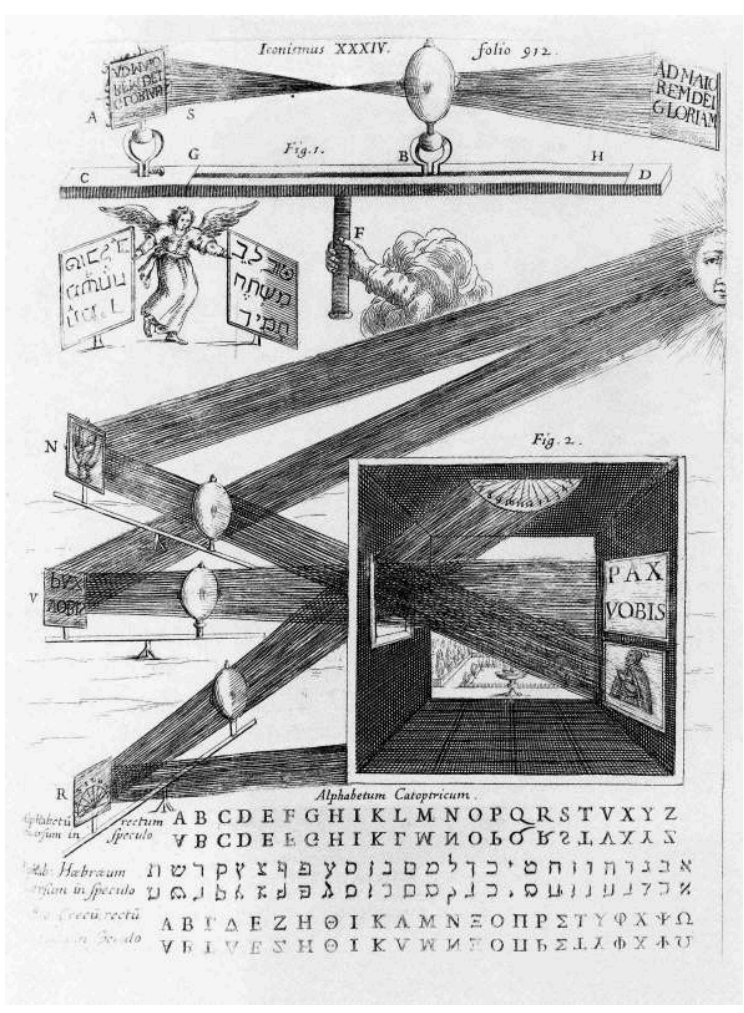

9 Fig. 3 - Projection d'inscriptions sacrées à travers des dispositifs optiques, dans : Athanasius Kircher, Ars magna lucis et umbrae, Rome, H. Scheus, 1646, p. 912, pl. 34 [source : Ich sehe was, was du nicht siehst! Sehmaschinen und Bilderwelten : die Sammlung Werner Nekes, Bodo von Dewitz und Werner Nekes, eds., Göttingen : Steidl, 2002, p. 178].

Dans une autre gravure provenant des Apiaria universae philosophiae mathematicae de Mario Bettini (1642), c'est la salle entière qui devient une caméra obscura, alors que la fenêtre est rendue complètement opaque. ${ }^{3}$ En pratiquant de petits orifices, la lumière est apportée via ces ouvertures, projetant les images mobiles sur le mur. La fenêtre a été aveuglée, et c'est justement cette obturation qui permet ce spectacle contrefait, « virtuel ». 


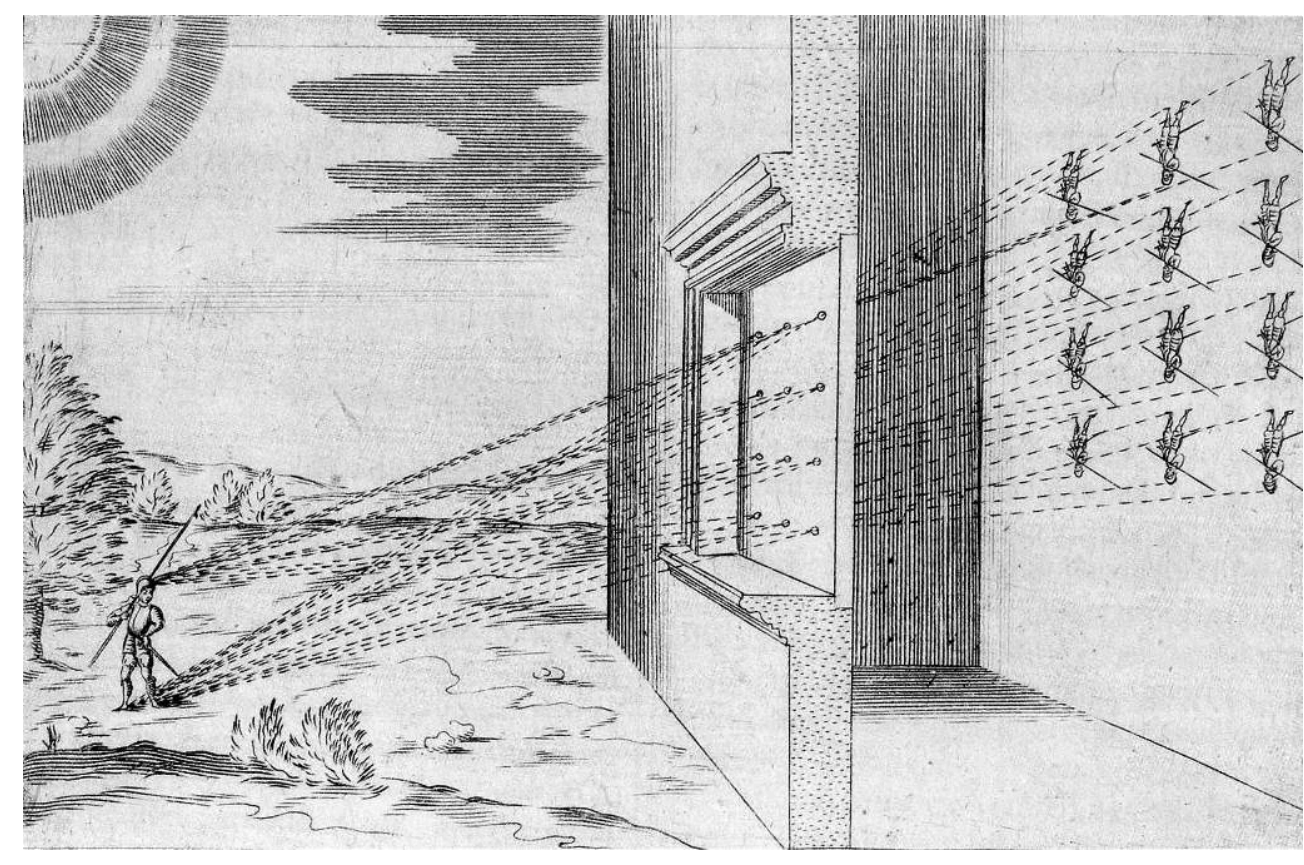

11 Fig. 4 - Camera Obscura, dans : Mario Bettini, Apiaria universae philosophiae mathematicae, Bologne, typis J. B. Ferronii, 1642, p. 38 [source : Ich sehe was, was du nicht siehst! Sehmaschinen und Bilderwelten : die Sammlung Werner Nekes, Bodo von Dewitz und Werner Nekes, eds., Göttingen : Steidl, 2002, p. 40].

La peinture-comme-fenêtre d'Alberti crée les conditions d'apparition du spectateur moderne, qui voit sans être vu. On peut regarder fixement par une fenêtre, et observer la vue ou les passants, mais sans être vu. La promotion du spectateur moderne est parallèle à l'apparition d'un regardeur armé de son "regard», précisément (ici, naturellement, on se rapporte à tout ce qui a été écrit sur le concept du regard). En conséquence, le monde représenté au travers de la fenêtre (perçu comme peinture) ne montre aucune continuité avec l'espace où l'observateur se tient: au lieu d'un continuum, il y a seulement une distance, qui est soulignée par le cadre de la fenêtre. Le regard du spectateur est asymétrique, parce qu'il regarde sans être vu. Ce manque de symétrie, qui apparaît pendant la Renaissance en même temps que la soi-disant « invention » de la perspective, deviendra un phénomène de plus en plus puissant, car il sera soutenu par de nouveaux dispositifs, tels que le Panopticon de Jeremy Bentham, par exemple. Cependant, la fenêtre Albertienne n'est pas simplement une ouverture vers un extérieur; elle définit beaucoup d'ensembles d'espaces opposés : l'extérieur et l'intérieur ; ce qui est illuminé par la lumière et ce qui reste dans l'ombre ; le visible et l'invisible, le manifeste et le caché. Comme Wacjman le précise, c'est par la peinturecomme-fenêtre que l'homme se libère d'un regard tyrannique et divin, «devenant de ce fait le Visionneur du Monde, d'un monde devenu image. » Avec cette idée de quelque chose de caché s'ouvre la possibilité de l'intime, un terme dérivé du latin intimus, le superlatif de l'intérieur, qui décrit ce qui est caché au regard des autres. L'intime est une notion plus forte que celle de "privé». La vie privée a été (et est toujours) principalement ordonnée et circonscrite par la loi. Elle est fondée sur la prohibition et le conseil : « il est interdit de regarder ou même de jeter un coup d'œeil, et, s'il vous plait, soyez discret". L'indiscrétion détermine un territoire fortement mutable, difficile à cartographier.

Chaque regard est indiscret, mais quand il pénètre les intérieurs domestiques, il devient intrusif, comme une violation, un viol, même. Pour un chrétien, laisser le 
regard pénétrer une maison correspond à un tel acte de violence. Se laisser regarder fixement revient un peu à ouvrir une maison comme si c'était un corps, laissant la corruption en franchir le seuil et en menacer l'âme à l'intérieur, l'âme à laquelle seule une puissance divine a accès. Les dangers de l'indiscrétion sont bien illustrés par une eau-forte de Cornelius Galle, éditée dans le Veridicus Christianus de Jean David (Anvers, 1601), dans laquelle une maison sous forme de tête humaine s'ouvre avec des fenêtres. ${ }^{4}$ La devise de la gravure avertit les lecteurs: "Adspectus Incauti Dispendium» (en allemand «Das Schauen gereicht dem Sorglosen zum Schaden»; en français « Regarder nuit à la personne négligente »).

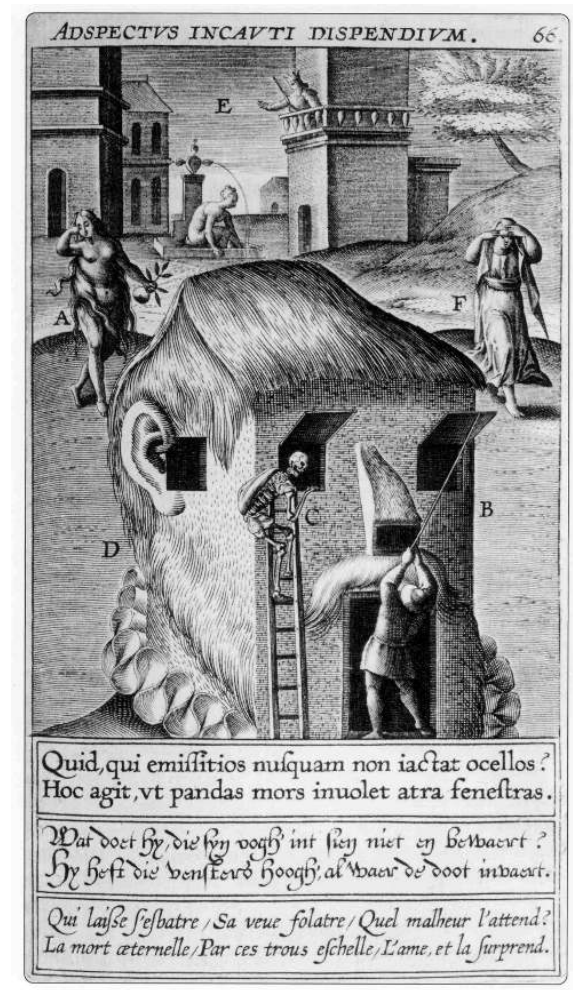

14 Fig. 5 - "Adspectus Incauti Dispendium," légende originale en latin, gravure de Cornelius Galle, dans : Jean David, Veridicus Christianus, Anvers, ex officina Plantiniana, 1601 [source : Die Wunderkammer des Sehens. Aus der Sammlung Werner Nekes,

Landesmuseum Joanneum, Graz, 2003, p. 47].

Cette illustration signifie qu'on doit comprendre que le corps lui-même est conçu comme maison. Menacé par des regards indiscrets et lourds de péchés, la maison doit être protégée. Comme un corps, le regard nocif pourrait s'infiltrer par la bouche (la porte), les narines (un hublot de ventilation), les oreilles et les yeux (fenêtres). Les prêtres et les moralistes de toutes les confessions y sont, eux, invités, pour protéger l'espace domestique avec tous ses orifices béants, y compris le sexe féminin. Le regard indiscret, celui qui s'immisce, peut mener l'observateur à la mort éternelle, ainsi qu'on le lit dans l'une des légendes multilingues de la gravure de Galle : « Qui laisse s'esbastre sa veue folatre / Quel malheur l'attend ? La mort aeternelle [sic]... ", écrit en latin, en vieil hollandais et en français archaïque. La condamnation répétée de la chair par les autorités religieuses aidera à dessiner une nouvelle géographie du caché, qui donnera une importance accrue à l'image des corps nus, et provoquera un nouvel érotisme, dont le voyeurisme sera une composante constante et active. Comme Wacjman le remarque, "Si on parle du voyeurisme [...], c'est parce que le corps lui-même est conçu comme une maison. 
Regarder à l'intérieur au travers des montants de la fenêtre pour entrer dans un corps interdit, de ce fait exécuter un acte fortement prohibé que chaque autorité essayait d'empêcher par tous les moyens. ${ }^{5}$ Tandis que le regard pénètre à l'intérieur, une nouvelle «jouissance » est née : regarder.

En regard de la progression de l'intimité, il semble nécessaire d'étudier l'origine de la délimitation entre les sphères publiques et privées, et comment leurs frontières ont été définies. Sans se référer à la longue histoire de ses sources philosophiques et légales, on peut peut-être se fonder sur un livre intitulé Bonheur privé, action publique(1982), où l'économiste et historien Albert Hirschman explique que le terme " privé » a en fait été soutenu comme un concept négatif, dérivé du mot latin privare, c'est-à-dire priver quelqu'un de quelque chose, déposséder quelqu'un de ce qu'il a. Ainsi c'était une notion (négative) de soustraction, par rapport à une autre, positive, qui était celle des affaires publiques, du bien commun. D'un point de vue historique, la privatisation a été un processus moins qu'évident. L'opinion innovante qui émerge néanmoins entre le $\mathrm{XVI}^{\mathrm{e}}$ et le XIX ${ }^{e}$ siècle, selon Hirschman, était que la poursuite d'intérêts privés constituait le but le plus probable pour assurer un ordre social harmonieux, comme cela avait été par exemple argumenté par le philosophe anglais Bernard Mandeville dans La fable des abeilles, ou Les fripons devenus honnêtes gens (1714). Même pendant le $\mathrm{XVIII}^{\mathrm{e}}$ siècle, un terme comme celui de «bonheur » avait toujours une dimension publique loin d'être négligeable. Au cours d'une telle période, les impératifs collectifs de la société s'étaient traduits dans la pratique de l'architecture par la théorie de l'embellissement urbain (urban beautification ou improvement en anglais). En fait, cette notion était un masque qui servit à la dissimulation des changements qui avaient lieu dans la ville. L'architecture "publique ", c'est-à-dire les bâtiments commissionnés par les membres de la famille royale ou par les princes, faisait partie de ce que les historiens ont nommé le «système du luxe. ». Le luxe délimitait une zone dans laquelle la dépense du roi ou du prince devait être manifestement affichée. Ainsi, c'est un secteur social que le luxe localisait dans la ville. L'ostentation de la richesse, qui définit l'espace même de son action, constitue ce qui pourrait s'appeler un réseau de monuments. Au XVIII ${ }^{e}$ siècle, le luxe était vraisemblablement le moyen le plus efficace d'établir l'ordre dans la société. ${ }^{6}$

Dans les villes de cette époque, on pouvait observer la contiguïté de deux espaces hétérogènes qui étaient opposés l'un à l'autre : d'un côté, le luxe ordonné ; de l'autre, le monde du chaos indistinct. Jusqu'aux années 1760 , l'architecture a lié son champ d'application à ce que l'on désignait alors comme le "luxe de bienséance ». ${ }^{7}$ En revanche, au XVIII ${ }^{\mathrm{e}}$ siècle, c'est le chaos qui a caractérisé l'espace privé. Cet espace a été considéré comme un lieu négatif par rapport au luxe, qui, lui, était le lieu des affaires publiques, le lieu de ce dont il était publiquement fait étalage, comme un spectacle. Ceci a été énoncé avec précision dans de nombreux textes relatifs à la théorie urbaine. Tous ces ouvrages formulaient un programme politique, ayant pour intention l'«amélioration» de la ville. Pour le résumer, le luxe et le chaos ont établi deux systèmes opposés au sein de la ville. Le monument présentait un spectacle répondant de ses propres règles, des règles qui pouvaient d'ailleurs être dictées par l'architecture, comme celles décrites dans le Cours d'architecture de Jacques-François Blondel [6 vol., 1771-1777] : « De la diversité des rangs \& de la dignité des sujets du Prince [...] doivent naître nécessairement les différents caractères [offrant] aux yeux des Étrangers \& des Citoyens, l'image des différents ordres d'un État policé. » [t. II, p. 237 $]^{8}$ Le privé n'a pas été administré selon ces théories du luxe. Au contraire, les théories avancées à son sujet 
ont été basées sur un système qui, au cours de cette période, a été décrit dans les traités édités par la police. Les constructions connues comme bâtiments privés, les édifices particuliers, ont formé le fond, l'arrière-plan indistinct de la ville. ${ }^{9}$ " Particulier » était d'ailleurs le terme employé au xvIII ${ }^{\mathrm{e}}$ siècle, en France, pour définir l'espace privé. Les «bâtiments privés » ont été tenus pour incontrôlables, et ont dû être régi non pas par des règles architecturales, artistiques ou esthétiques, mais dans des ouvrages de police, tel le célèbre Traité de la police (Paris, 1705-1738, 4 vol.) de Nicolas de La Mare. La césure entre deux régimes différents apparaît tout à fait clairement: d'une part, les édifices publics qui ont peu besoin de règlements, parce que les arts libéraux les ordonnent déjà ; d'autre part, les bâtiments privés, qui n'appartiennent pas au domaine de l'architecture, et qui requièrent une gouvernance intensive par la police.

Étymologique, bien sûr, le terme "police » est dérivé de la notion de polis, le mot grec pour ville. Cependant, entre la polis idéalisée dans toutes les discussions sur l'architecture et l'urbanisme, et la "police» et ses règlements, cette police soupçonneuse, prudente, quelques relations singulières ont été créées. Il y a toujours un côté sombre de l'urbanisme que les historiens ne devraient jamais oublier de prendre en considération. Après tout, l'urbanisme, comme l'hygiène, a été inventé pour apporter de l'ordre à ce qui était désordonné. En outre, ces dualités, ces oppositions, avaient déjà reçu un soutien théorique au XvIII ${ }^{\mathrm{e}}$ siècle. La distinction entre les concepts de monumental et de particulier constitue une sorte d'archéologie de la division entre les espaces publics et privés, ainsi que cela sera établi durant le XIXe siècle.

Comme noté auparavant, la fenêtre fonctionne toujours selon une nature double, l'une concernant le bâtiment, l'autre reliée au système optique. L'aspect architectural est basé sur des besoins, bien qu'il n'exclu pas l'usage optique de la fenêtre. Il semble cependant que, à l'exception d'une utilisation à but militaire, l'usage «optique " des fenêtres ne faisait pas partie de la théorie ni de la pratique architecturale. Il peut être soutenu, comme Wacjman le maintient, que la fenêtre architecturale est aveugle, et que le regard n'est pas l'objet principiel de l'architecture, mais celui de la peinture. Sans contester une telle affirmation (même si on le pourrait), il serait intéressant de vérifier si la connaissance architecturale qui s'applique aux façades des palais, de la première Renaissance italienne jusqu'à la période Baroque, était toujours capable d'éluder des questions telles que la division horizontale des étages avec le piano nobile, la répétition rythmée d'arches et d'édicules, le choix des ordres des colonnes ou des pilastres, etc. ${ }^{10}$ $\mathrm{Du}$ fait que l'architecture appartient à un régime du luxe qui s'expose afin d'imposer un ordre à la société, les bâtiments se donnent à voir par leurs surfaces extérieures. En tant qu'objet visible, on parle de la façade, ou même du « visage » d'un bâtiment vers la rue, visage qui a été défini par André Félibien comme : « le côté de devant par où l'on y entre, ou une partie considérable qui se présente à celui qui le regarde ${ }^{11}{ }^{11}$ Puisque l'architecture se rapporte à l'image d'un corps humain, au lieu d'une façade, il serait plus précis de faire allusion à un visage; même si ce serait un visage exempt d'un regard.

Puisqu'il n'y a aucun œil qui regarde, les façades percées par des rangées des fenêtres sont comme un visage sans regard, ce qui signifie que la fenêtre architecturale est aveugle. Une représentation typique de ces fenêtres aveugles est offerte dans les gravures de Jean Marot représentant l'Hôtel de la Vrillière, une résidence aristocratique édifiée par François Mansart à Paris de 1635 à $1650 .{ }^{12}$ 


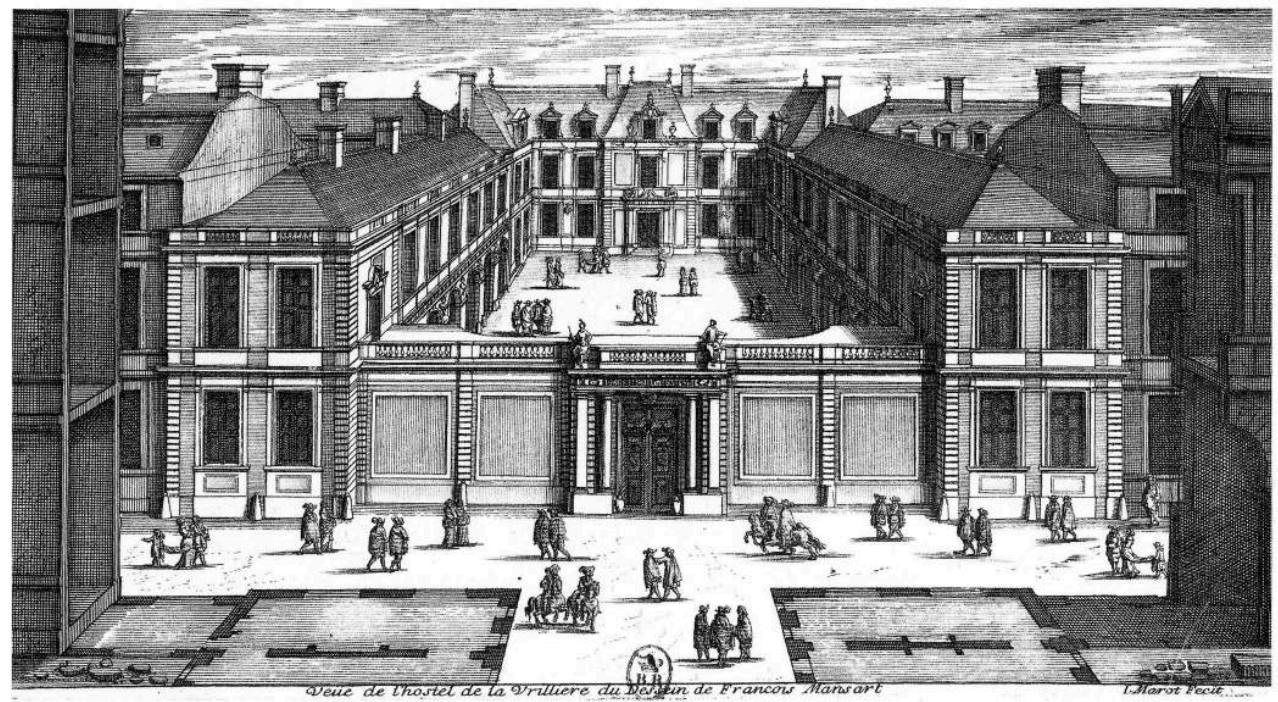

Fig. 6 - « Vue perspective de l'Hostel de la Vrillière du dessein de François Mansart »,

Hôtel de la Vrillière, Paris, de 1635 à 1650, gravure de Jean Marot, (BN, Estampes,

Paris) ; [source : François Mansart : le génie de l'architecture, éd. Jean-Pierre Babelon,

Claude Mignot, Paris, Gallimard, 1998].

Naturellement, on a toujours pu regarder par la fenêtre. Néanmoins, ceci signifie qu'en vertu de la condition de la fenêtre on devrait distinguer une fenêtre optique et une fenêtre architecturale. Il faut essayer de différencier deux régimes scopiques, et ce qui les rendent dissemblables. Au cours de la période classique et moderne, la fenêtre architecturale a souvent ressemblé au visage aveugle du pouvoir; elle le restera d'autant plus pendant le $\mathrm{xx}^{\mathrm{e}}$ siècle, avec l'introduction du mur rideau vitré de l'immeuble de bureaux

Derrière la conception victorienne (ou haussmannienne) du privé et du public se présente une topographie sur laquelle l'existence juridique de l'habitation a été basée. Ainsi les seuils, abrités par une série de gardiens et portiers, deviennent des filtres complexes, des tamis qui laissent l'extérieur venir à l'intérieur, et vice-versa. En France, par exemple, la première disposition légale qui a assuré la non-violabilité du domicile a été votée en 1791 (art. 184, code pénal). Pendant la première moitié du XIX siècle, l'expression "mur de la vie privée " assumera sa signification légale et sociale. Le terme français "intérieur » ne se réfère plus à l'intériorité du cœur ou de l'âme humaine, mais à ce qui garantit en son sein les conditions pour le bonheur et le confort. Néanmoins, peu de temps après, les nouveaux médias de communication - livres, journaux et magazines, etc.- entreront dans les espaces domestiques, et une connaissance planétaire pénétrera ces salles calmes, avant même la diffusion de nouvelles technologies telles que le téléphone et l'électricité. ${ }^{13} \mathrm{En}$ fait, puisque la maison devient l'endroit de passions contradictoires, elle apparaîtra, d'un côté, comme la forteresse de l'«intimité " (la notion anglaise de "privacy», ou celle de " Gemütlichkeit » en allemand), alors que, de l'autre, elle se présentera comme un microcosme quadrillé de frontières mobiles. Dans les espaces domestiques, le publique et le privé s'affrontent ; un état de guerre qui mènera à l'exclamation célèbre d'André Gide : «Familles, je vous hais! Foyers clos; portes refermées; possessions jalouses du bonheur. ${ }^{114}$ La ligne de démarcation entre les sphères publiques et privées forme un champ de bataille actif, se modifiant et se réajustant constamment à mesure que les normes de la 
société changent et évoluent. Pour exemple, le téléphone est perçu comme une infraction insupportable par un certain "homme difficile» (dans la comédie d'Hofmannsthal, Der Schwierige), alors que, dans le même temps, il permet que des informations personnalisées et individualisées soient acheminées jusque dans l'espace privé. ${ }^{15}$

Cependant, les seuils ne constituent que rarement une barrière à l'espace public, si tant est qu'ils ne l'aient jamais été ; pas plus qu'ils ne sont conçus comme un obstacle à la perméabilité. Probablement, pour trouver des indices quant à cette condition de limite, doit-on relire l'essai de 1909 de Georg Simmel sur Le pont et la porte (Brücke und Tür), où il affirme que la nature humaine est composée tant de séparation que d'unification, comme les deux berges d'une rivière, qui sont séparées seulement pour être réunies par un pont. ${ }^{16}$ De façon semblable, un être humain est une créature limitrophe, vivant en permanence sur la frontière, bien que n'ayant aucune limite pour se circonscrire luimême. La porte procure les moyens pour contenir l'être (humain), mais elle est également ce qui permet de sortir, en traversant la frontière. Ici Simmel développe un de ses sujets préférés, qui est toujours la base pour établir un réseau infini de relations réciproques, ou d'interactions (en allemand, Wechselwirkung), l'un de ses concepts fondamentaux. L'état réciproque d'unité/exclusion avait déjà été tracé par l'essai de 1902 de Simmel sur Le cadre: une étude esthétique (Der Bildrahmen. Ein ästhetischer Versuch), une recherche éclairante sur ce qu'implique le fait d'encadrer un tableau. ${ }^{17} \mathrm{Ici}$, le cadre, par la matérialité même de la frontière qu'il institue, est pensé comme un dispositif qui aide la peinture à exclure quoi que ce soit d'extérieur, tout en permettant la concentration en son intérieur. Le cadre isole l'œuvre d'art, en interdisant la possibilité d'un commerce perpétuel avec l'extérieur, tels que des phénomènes biologiques le permettraient (comme l'exosmose et l'endosmose dans une cellule). Pour Simmel, la position du cadre est secondaire par rapport au tableau, et rien ne devrait venir lutter contre cela ; bien au contraire, tout (la matière, la couleur, la façon et la forme) devrait contribuer à maintenir sa nature de Parergon (en grec, un travail subalterne, ou un accessoire), un écho probable aux considérations de Kant sur le statut de l'œuvre peinte (en grec, Ergon, le travail principal). Toute tentative d'affaiblir l'essence de l'encadrement, que ce soit en ouvrant cette frontière qui cerne l'œuvre, en transformant le cadre en élément de mobilier, ou en utilisant la forme d'éléments architecturaux (tels que des pilastres ou des frontons) pour établir le cadre, est condamnée, puisqu'elle diminuerait son caractère de parergon.

Ceci se produit parce que le cadre, tout comme la porte, se trouve en position instable, agissant en tant qu'intermédiaire entre la peinture et ses environnements - auxquels elle est unie et dont elle est séparée en même temps. Pour Simmel, le cadre d'une peinture doit être pensé comme la métaphore d'une frontière entre des groupes sociaux; montrant, ainsi, que la vraie fonction de l'encadrement est de "fermer» l'objet «contre le monde environnant et de maintenir son unité. $»^{18}$ Dans son essai sur le cadre du tableau, Simmel assimile l'intégrité simultanée d'une œuvre d'art en soi et comme un tout unifié avec ses environnements, aux difficultés rencontrées par les entités sociales collectives, qui ont ces aspirations contradictoires d'appartenir à un groupe, et néanmoins de se revendiquer comme étant des individus autonomes. En conclusion, l'œuvre de Simmel propose une théorie complexe du passage et de la frontière qui influencera ses élèves, tels que Siegfried Kracauer, mais également le Walter Benjamin des images dialectiques. Pour Simmel comme pour Benjamin, la frontière, qu'elle soit limite ou démarcation, se révèle à travers une condition 
médiatrice, comme un "milieu» au sens littéral du terme. Chaque seuil, ou zone marginale, induit une condition réciproque.

À la fin du XIX siècle, avec l'électrification des maisons et l'embranchement des lignes téléphoniques, c'est l'énergie elle-même qui envahit l'intérieur. La transparence n'est pas tant affaire de l'utilisation du métal et du verre dans la construction, mais bien plus la question de la façon dont les énergies seront figurées et représentées : d'où les concrétions de la force vitale, engendrées par la «ligne force» chez Henry van de Velde, qui intègre des facteurs invisibles tels que les vibrations ou les rayons X. Autour de 1900, le concept d'éther était compris comme ce médium impondérable qui emplirait entièrement l'espace et véhiculerait les vibrations des ondes électromagnétiques. Dans l'imagination populaire, l'éther était synonyme d'espace. ${ }^{19}$ L'intérieur est maintenant un lieu perméable où les corps peuvent agir dans les champs de force. Dorénavant, le périmètre matérialisé par l'architecture devra pourvoir tant à la séparation qu'à la perméabilité, devenant ce que la biologie a nommé une membrane.Dans la cellule, une telle surface est ce qui permet à l'organisme de trouver un équilibre entre la solidité et la perméabilité (c'est-à-dire un équilibre des fluides), assurant les conditions pour des processus tels que l'osmose. Dans ce type de logique, Siegfried Ebeling, un étudiant du Bauhaus, a édité, en 1926, un volume intitulé Der Raum als Membran (L'espace comme membrane), dans lequel il présente son idée d'une maison conçue comme un espace multicellulaire, creux, dont les surfaces, exemptes de tout ce qui empêcherait le flux des énergies, pourraient permettre les processus du métabolisme. ${ }^{20}$

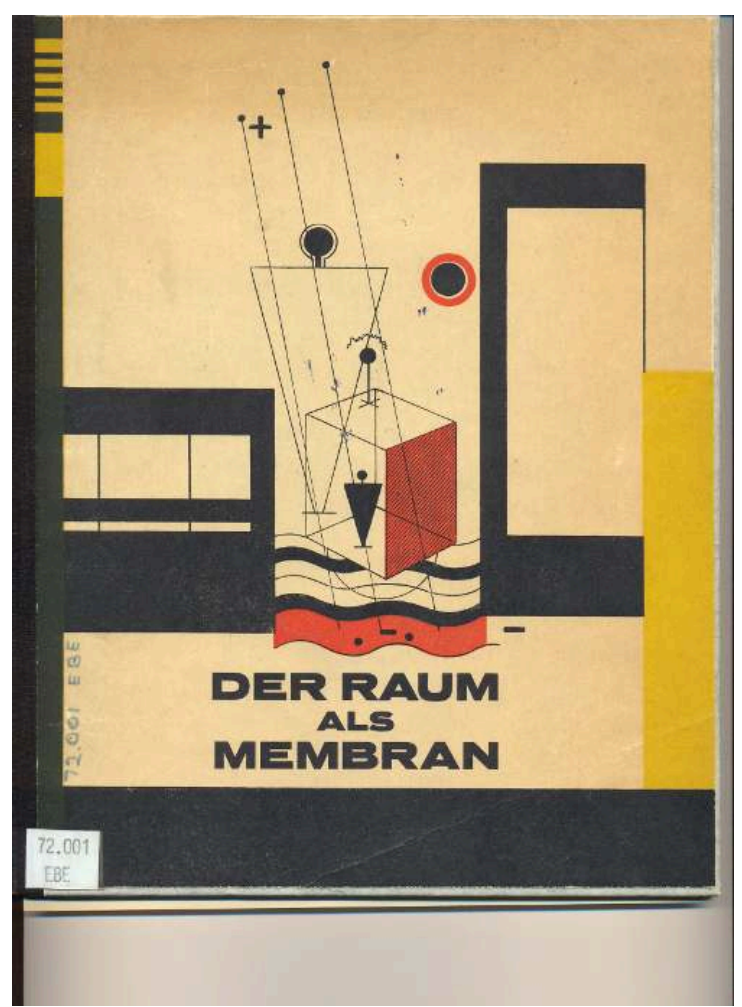

Fig. 7 - «L'espace comme membrane », couverture de l'ouvrage : Siegfried Ebeling, Der Raum als Membran ist ein analytisch-kritischer Beitrag zu Fragen zukünftiger Architektur, die über das nackte Bedürfnis hinausgeht und hiermit sich legen möchte in die gestaltende Hand aller Wissenschaft, Dessau: Dünnhaupt, 1926. 

élément, comme Aristote l'a appelé), et, en incarnant les forces invisibles, elle acquiert un caractère éthéré. Ce n'est pas un hasard si le nom donné par Bob Metcalf aux réseaux informatiques locaux (LAN) à Xerox PARC en 1973 était "Ethernet», une invention qui régira la base de la communication dans notre existence technologiquement appareillée, propageant nos pensées, sentiments et désirs à une échelle planétaire. ${ }^{21}$ Ces mêmes années, Gilles Deleuze lui-même s'était approprié une théorie biologique de l'individuation et de la différentiation en lisant les travaux magistraux de Gilbert Simondon sur la philosophie des sciences et de la technologie, dont les volumes l'aideront à définir la notion du «corps sans organe», en ancrant la base de ce concept dans la biologie.Dans son travail, Deleuze avance une théorie des limites, qui aide à surmonter le dualisme entre intériorité et extériorité, et lui permet de soutenir que la peau est la chose plus profonde : «La vie vit à la limite d'elle-même, sur sa limite... Les polarités caractéristiques de la vie sont situées au niveau de la membrane. $»^{22}$ Dans sa description des processus de l'individuation, Simondon réussit à explorer les strates pré-individuelles comme source des singularités. Un individu, selon Simondon et Deleuze -, n'est pas un être stable, mais le résultat des processus, des opérations et des formes, une confluence d'énergies différentielles, une rencontre entre affects, percepts et émotions.

29 C'est la notion même de limite qui doit être remise en cause. Selon Paul Virilio, dans L'espace critique(1984), les limites sont actuellement définies par l'interface entre l'homme et la machine, non plus par les façades des bâtiments, ni par les surfaces des parcelles de terrain. La conception précédente du couple privé/public, et la différentiation entre la maison et la rue, est remplacée par une nouvelle «topologie électronique ", en ce que la grille de l'image numérique supplante les divisions plus anciennes, telles que la division des parcelles urbaines, lui substituant une surexposition qui annule la distinction clairement définie entre proximité et distance. L'étude de l'interface homme/machine a mené à la généralisation de la cybernétique. $\mathrm{Du}$ contrôle des machines par des systèmes autorégulateurs (comme la servocommande) jusqu'aux considérations sur la configuration du corps comme machine informationnelle, nous habitons des systèmes interactifs, suscitant des réponses sensorielles au sein d'environnements virtuels, capables de recréer le monde et donc de générer les sites d'une intersubjectivité inédite, tant motrice que émotionnelle.

À présent, nos fenêtres sur le monde semblent être ces écrans multiples, portatifs et nomades : la télévision avec des écrans à cristaux liquides ou des vidéo projecteurs, les ordinateurs portables, les caméscopes, les appareils photo numériques, les appareils photo des téléphones sans fil, les organiseurs (PDA pour Personal Digital Assistant)et les smart phones, les écrans tactiles, les lecteurs MP3, les écrans de GPS, le téléchargement de balados (podcasts), les consoles de jeu interactives dotées d'un détecteur de mouvement. À l'heure actuelle, comme Bill Moggridge l'indique : «Les concepteurs de produits technologiques numériques ne considèrent plus leur tâche comme la conception d'un objet physique, [...], mais comme la planification des interactions de l'utilisateur.» Aujourd'hui, les téléphones portables montrent leur mauvais "visage », qui se révèle au travers de leur niveau de complexité et d'incohérence. Selon les concepteurs, l'aspect rébarbatif affiché par certains téléphones portables résulte du nombre élevé des intervenants dans ce secteur, ce qui contribue à la fragmentation des 
infrastructures de réseaux, à la vaste panoplie des combinés, aux différentes interfaces en conflits, et aux problèmes de mise à l'échelle de l'accès à Internet et au Web. ${ }^{23}$

31 La musique s'écoule au travers de l'éther jusque dans des machines minuscules et élégantes: ces appareils font-ils plus de mal que de bien? De telles technologies améliorant les sens, de l'iPod à l'Internet sans fil, sont-elles les parties émergées, les affleurements évidents d'un bouleversement total des façons dont le monde est encadré? Les stratégies industrielles et étatiques de contrôle sont tout à fait antipathiques, toutefois l'artifice pourrait offrir les moyens d'ouvrir quelques portes à la perception. Du côté positif, on peut assister à des festivals de vidéos produits par les appareils photographiques des téléphones mobiles. Pour le négatif, au travers de la technologie invasive des téléphones portables, on est submergé par les ennemis acoustiques. D'un autre côté, de grands succès ont été réalisés avec les gadgets audio portatifs, parce qu'ils permettent de manipuler les sons, qui sont très proches de nous et de nos pensées, affects et percepts. Le son se déplace littéralement au travers du corps. Les casques et écouteurs ont créé un univers auditif privé.Néanmoins, la promulgation d'une privatisation auditive procure également les ingrédients pour une situation contradictoire, un champ de bataille sur lequel le son, la musique et le bruit lutteront. Par sa capacité à empiéter, la technologie des téléphones portables invite à la prestation publique de sons "privés». Comme le note Michael Bull, «Les téléphones portables sont exemplaires, confondant la séparation des sphères publiques et privées et le rapport souvent contradictoire et changeant que nous entretenons avec elles. $»^{24} \mathrm{En}$ conséquence, la seule défense contre le bruit n'est pas le silence, mais plus de bruit. En outre, du fait que les technologies audio numériques deviennent de plus en plus ambitieuses, les espaces domestiques sont, conséquemment, de plus en plus divisés en de multiples modules d'écoute et de visualisation.

32 Tant que l'on mentionne les dispositifs audio, on pourrait reconnaitre que l'iPod d'Apple est la première icône culturelle du consommateur du xxi siècle. Non seulement le design de l'iPod est raffiné et simple - et même simplet -, mais le logiciel d'interface utilisateur, créé par l'équipe de Paul Mercer (fondateur de Pixo), est encore plus intelligent. Successeur du baladeur de Sony, le dispositif élégant propose des sphères soniques privées, que révèle le principe de liste d'écoute personnelle. Dans sa miniaturisation, la technologie acquiert une touche de magie, casant un vaste monde numérique dans le creux de la main. Lorsqu'ils portent leur propre juke-box personnel, les utilisateurs sont entourés d'une acoustique qui les enveloppe, et ils se déplacent en rythme dans l'espace de la ville, drapés dans leurs bulles auditives. Bien que l'on soit continuellement agressé par du bruit parasite, l'instrument portatif a créé une zone mobile d'immunité et de sécurité. ${ }^{25}$ Les appareils nomades de notre mode de vie sont doués d'une vie propre fascinante, prolongeant probablement les contours de nos sphères égotistes, exécutant des expériences dans lesquelles le corps est le nœud principal pour la médiation technologique. Dans un même ordre d'idée, l'animation du corps va désormais dépendre des multiples connections, à la fois prosthétiques et esthétiques, reliant entre elles des machines fabriquées autour de semi-conducteurs en silicium, ce qui pourrait engendrer un nouveau type de phylum, une phylogenèse insolite dont on n'a pas terminé de répertorier les embranchements. ${ }^{26}$

33 Nous sommes entourés par ces dispositifs portatifs envahissants, qui transforment profondément notre mode de vie. Le philosophe Jean-Louis Déotte distingue trois étapes principales dans le processus de telles mutations technologiques. Ces phases 
sont dénommées d'après l'œuvre de trois penseurs majeurs $d u x^{e}$ siècle: Benjamin, Michel Foucault et Deleuze. Les trois paradigmes consistent, respectivement, dans le dispositif prothétique, le régime de contrôle, et le flux nomade. Aucun d'eux n'a une existence (ou une explication) chronologique, pas plus qu'ils ne montrent une logique diachronique significative; simplement, ils coexistent dans nos sociétés postutopiques.

Tout d'abord, avec Benjamin, on peut considérer une théorie de l'appareil (photo et cinéma), qui révèle comment le cameraman adopte la position d'un chirurgien, puisque les deux opérateurs mettent en application des pratiques qui, d'un point de vue conceptuel, sont proches de la nature d'un équipement prothétique. Pour Benjamin, comme avec L'homme à la caméra de Dziga Vertov (1929), «le caméraman peut pénétrer la réalité avec l'appareil. $»^{27}$ Aujourd'hui, l'appareil porté par l'artiste Hiroo Iwata, son điil flottant (2000), propose une illustration adéquate d'une telle prolongation prothétique de l'œil. ${ }^{28}$

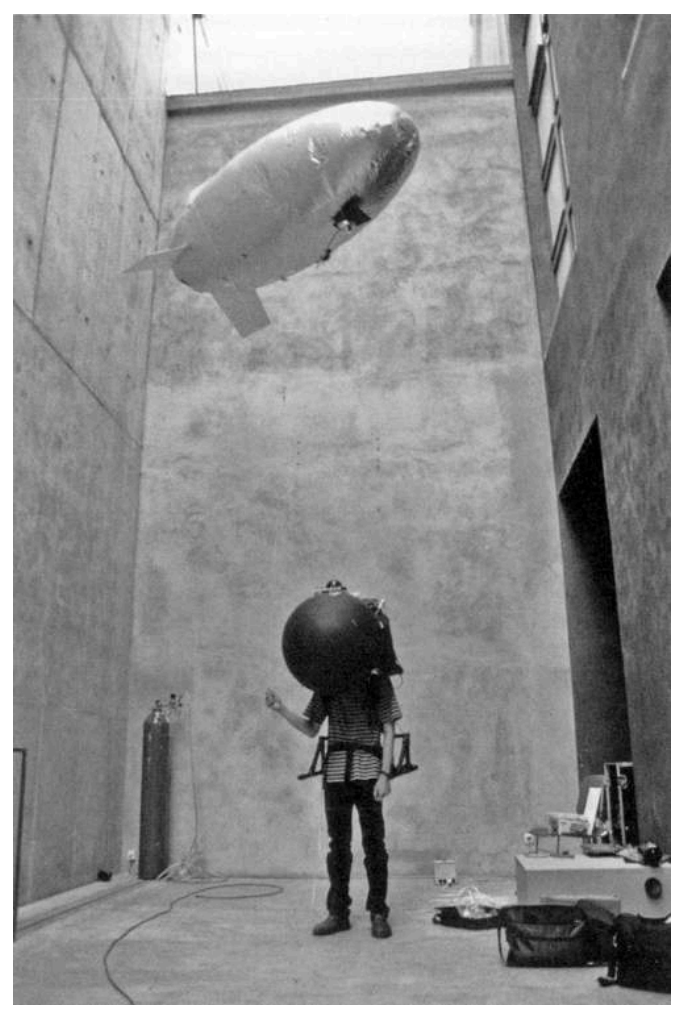

Fig. 8 - Hiroo Iwata, Eil flottant / Floating Eye, 2000, Ars Eletronica [source : Oliver Grau, Virtual Art. From Illusion to Immersion, Cambridge, MA, The MIT Press, 2003].

Deuxièmement, avec la théorie du dispositif de Foucault, qui a choisi le Panopticon de Bentham comme l'un de ses épitomes, on est confronté à une archéologie de la fenêtre optique, qui permet l'articulation de deux séries hétérogènes: celle du savoir en sciences sociales, et celle du pouvoir, en examinant, par exemple, les règles de la vision et les différents régimes de la visualité de diverses hétérotopies. L'exposition de 2002 sur les Rhétoriques de Surveillance au ZKM, le centre pour l'Art et les Médias de Karlsruhe, a offert une illustration fascinante du sujet. ${ }^{29}$ Plus récemment, Giorgio Agamben a montré que le concept de dispositif appartient au domaine de l'économie dans sa signification Aristotélicienne, que le premier catholicisme et la scolastique s'approprieront. Pour Agamben, n'importe quel dispositif (y compris la confession, la 
pénitence, la prison et ... les téléphones portables) mène à un processus d'assujettissement inexorable. Ainsi, " [Un] dispositif est donc, avant tout, une machine qui produit des subjectivations et c'est par quoi il est aussi une machine de gouvernement. ${ }^{30}$

Troisièmement, les concepts de machines désirantes et de disposition nomade sont exposés par Deleuze (avec Félix Guattari) dans le célèbre chapitre XII de Mille plateaux. ${ }^{31}$ En conséquence, les sujets nomades sont libres de flâner et de vagabonder à travers des espaces unis (non striés) et lisses, où aucune limite aprioristique ou artificielle, où aucune barrière, n'ont été tracées sur la terre. La libération des flux et des circulations qui s'opère dans le mouvement permet de quitter le vieux paysage, en ouvrant un nouveau territoire. De plus, la réalité nomade offre les conditions d'un déplacement à grande vitesse, même si l'on reste immobile. Le meilleur exemple, peut-être, pour montrer un tel état nomade a été l'installation de 1985 de Toyo Ito, intitulée Un abri pour femmes nomades de Tokyo.

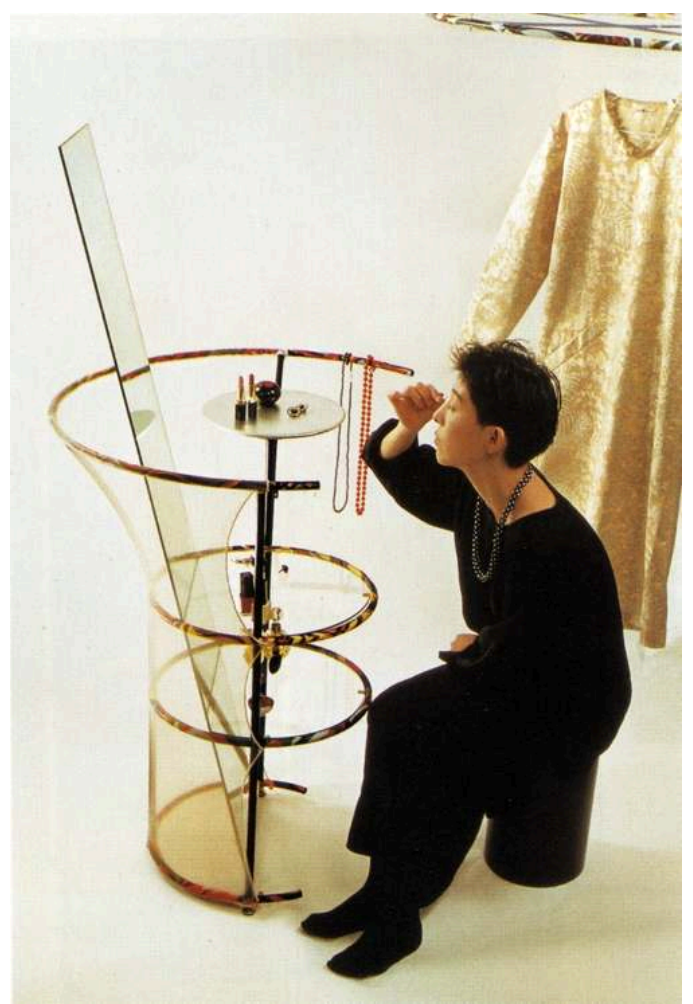

38 Fig. 9 - Toyo Ito, Projet pour PAO 1, « Abri pour femmes nomades de Tokyo ", installation, Grand magasin Seibu, Tokyo, 1985 [source : Works, Projects, Writings: Toyo Ito, Andrea Maffei, ed., London \& Milano, Blackwell and Electa, 2002.]

Pour Deleuze, "les nomades sont toujours au milieu [...]; [ils] n'ont ni passé ni avenir, seulement des devenirs. [Ils] n'ont pas d'histoire, [...] seulement de la géographie.» ${ }^{32}$ L'accent qui était porté sur la temporalité s'est transformé en question de spatialité, tandis qu'une conscience planétaire s'est accrue et développée, devenant topographique et, avec l'augmentation des réseaux, même topologique.

Aujourd'hui, notre expérience quotidienne est effectivement caractérisée par la notion d'un virtuel, « la réalité ambiante », une expression qui évoque la capacité de rester en contact avec des personnes de façon régulière en utilisant différents sites Web, une pratique sociale qui aide à vivre sa connexité. ${ }^{33}$ Par la télévision, le câble, l'Internet, et d'autres canaux, on prend part à une progression de l'érotisation des relations sociales, 
au moins dans l'hypothèse du sociologue Michel Maffesoli, inspirée par le travail de Simmel. ${ }^{34}$ Le succès des émissions de télé-réalité et des sites en ligne (MySpace, Facebook, YouTube, Second Life, Twitter) révèle une passion à se donner en spectacle, marquant ainsi un nouveau seuil, qui mène de l'ère de l'intimité à celle de l'« extimité $» .^{35} \mathrm{En}$ capturant les moments fugaces entre exhibitionnisme et voyeurisme, les photographes comme Merry Alpern ou les cinéastes tels que Nicholas Barker ont présenté dans leurs images la pan-érotisation en cours du monde. ${ }^{36}$ Néanmoins, au lieu d'une progression de l'individualisme, que certains s'accordent à lier à l'utilisation d'Internet, quelques sociologues trouvent de nouveaux types d'attraction et d'attachement, capables de solidifier des groupes, tout en croisant de vieilles barrières, un phénomène qui pourrait être qualifié de néo-tribalisme. Une fois de plus, ce qui semble en mesure d'émerger serait une série d'interactions, lourdes de motivations dionysiaques qui apporteront des effets sociaux puissants, capables de mener à une densification de la vie quotidienne, restituant peut-être à la notion d'esthétique sa signification originale, celle des émotions partagées.

\section{NOTES}

1. Gérard Wajcman, Fenêtre: chroniques du regard et de l'intime, Lagrasse : Verdier, 2004, p. 19-21, 35.

2. Anne Friedberg, The virtual window: from Alberti to Microsoft, Cambridge, Mass.: MIT Press, 2006, p. 26-42; and 103.

3. Ich sehe was, was du nicht siehst!: Sehmaschinen und Bilderwelten: Die Sammlung Werner Nekes, [Ausstellung, Museum Ludwig, Köln, 27. September bis 24. November 2002], hrsg. von Bodo von Dewitz und Werner Nekes, Göttingen: Steidl, 2002; Eyes, lies and illusions: the art of deception: drawn from the Werner Nekes collection, [exhibition, London, Hayward gallery, 7 October 2004 - 3 January 2005], Laurent Mannoni, Werner Nekes, Marina Werner, London: Hayward gallery ; Aldershot: Lund Humphries, 2004.

4. Die Wunderkammer des Sehens: aus der Sammlung Werner Nekes, [Ausstellung Landesmuseum Joanneum, 7. November 2003 bis 21. März 2004], Graz : Landesmuseum Joanneum, 2003, p. 46.

5. Wajcman, Fenêtre..., op. cit., p. 372, 374-376, 439-440, 447, 450.

6. Jean-François Harouel, L'embellissement des villes. L'urbanisme français au XVIII siècle, Paris, Picard, 1993 ; Philippe Perrot, Le luxe. Une richesse entre faste et confort, XVIII ${ }^{e}$ XIX ${ }^{e}$ siècle, Paris, Seuil, 1995.

7. Anne Röver-Kann, Bienséance : zur ästhetischen Situation im Ancien Régime, dargestellt an Beispielen der Pariser Privatarchitektur, Hildesheim ; New York : G. Olms, 1977 ; Bettina Maria Köhler, Die Stadt Paris und das Wohnhaus: zum "Bâtiment Particulier" in der französischen Architekturtheorie von 1600-1750, Alfter : Verlag und Datenbank für Geisteswissenschaften, 1994.

8. Natacha Coquery, L'hôtel aristocratique : le marché du luxe à Paris au XVIII siècle, Paris, Sorbonne, 1998 ; Id., L'espace du pouvoir : de la demeure privée à l'édifice public, Paris, 1700-1790, Paris, S. Arslan, 2000.

9. Jean-François Cabestan, La conquête du plain-pied: l'immeuble à Paris au XVIII siècle, Paris, Picard, 2004. 
10. Elisabeth Heil, Fenster als Gestaltungsmittel an Palastfassaden der italienischen Früh- und Hochrenaissance, Hildesheim ; New York : G. Olms, 1995 ; Sabine Lietz, Das Fenster des Barock: Fenster und Fensterzubehör in der fürstlichen Profanarchitektur zwischen 1680 und 1780, Munich: Deutscher Kunstverlag, 1982.

11. André Félibien, sieur des Avaux, Des principes de l'architecture, de la sculpture, de la peinture, et des autres arts qui en dépendent, Paris : J. B. Coignard, 1676 ; reprint, Farnborough (Hants), Gregg, 1967, p. 187.

12. François Mansart: le génie de l'architecture, Jean-Pierre Babelon, Claude Mignot, eds., Paris, Gallimard, 1998

13. Histoire de la vie privée, Philippe Ariès, Georges Duby, eds., Paris, Seuil, 1985-1987 ; vol. 4. De la Révolution à la Grande Guerre, Michelle Perrot, ed., Seuil, points histoire, 1999, n² 263, p. 15, 281, 284-286, 563.

14. André Gide, Les Nourritures terrestres, Paris, Mercure de France, 1897, Livre IV.

15. Hugo von Hofmannsthal, Der Schwierige [1921], ed. W. E. Yates, Cambridge, UK: Cambridge University Press, 1966.

16. Georg Simmel, "Brücke und Tür”, in Id., Das Individuum und die Freiheit. Essais, Frankfurt a. M.: Fischer Taschenbuch Verlag, 1994, p. 2-12 ; Id., "Pont et porte", in La tragédie de la culture: et autres essais, Paris, Rivages, 1993, p. 159 ; Georg Simmel, “Bridge and Door [1909]”, translated by Mark Ritter, Theory, Culture \& Society, 1994, 11:5-10.

17. Georg Simmel, "Der Bildrahmen. Ein ästhetischer Versuch" [1902], in: Id., Gesamtausgabe, Otthein Rammstedt, éd., Francfort-sur-le-Main, Suhrkamp, t. 7, (Aufsätze und Abhandlungen 1901-1908), [vol. I], 1995, p. 101-108 ; Id., « Le cadre : un essai esthétique », in : Id., Le cadre et autres essais, trad. Karine Winkelvoss, Paris, Gallimard, 2003, p. 29-40 ; Georg Simmel, "The Picture Frame: An Aesthetic Study”, Theory,Culture \& Society, (1994), 11(1):11-17.

18. Georg Simmel, “Soziologie des Raumes”, Aufsätze und Abhandlungen, 1901-1908, Frankfurt a. M.: Suhrkamp, 1995, p.132-83: “[...] gegen die umgebende Welt ab- und in sich zusammenzuschließen" (p.138); Id., "The Sociology of Space”, in: Simmel on Culture: Selected Writings, David Frisby, Mike Featherstone, eds., London: Sage Publications Inc., 1997, p. 137-170, p. 141.

19. Linda Dalrymple Henderson, "Vibratory Modernism: Boccioni, Kupka, and the Ether of Space", in: From Energy to Information: Representation in Science and Technology, Art, and Literature, Bruce Clarke, L. Dalrymple Henderson, eds., Stanford, Calif.: Stanford University Press, 2002, p. 126-149.

20. Christoph Asendorf, "Bodies in Force Fields: Design between the Wars", in: From Energy to Information..., op. cit., p. 195-212.

21. Caroline A. Jones, "ether", in: Sensorium: Embodied Experience, Technology, and Contemporary Art, C. A. Jones, ed., Cambridge, Mass.: MIT Press; The MIT List Visual Arts Center, 2006, p. 137-141.

22. François Dosse, Gilles Deleuze, Félix Guattari. Biographie croisée, Paris, La Découverte, 2007, p. 200-201; Deleuze cite : Gilbert Simondon, L'individu et sa genèse physico-biologique, Paris, PUF, 1964 , p. 260.

23. Bill Moggridge, Designing interactions, Cambridge, Mass.: MIT Press, 2007, p. 315-316, p. 390.

24. Michael Bull, “auditory”, in: Sensorium, op. cit., p. 112-114.

25. M. Bull, "iPod”, in: Sensorium, op. cit., p. 156-158.

26. C. A. Jones, "The Mediated Sensorium", in: Sensorium, op. cit., p. 5-49, esp. p. 43.

27. Jean-Louis Déotte, Qu'est-ce qu'un appareil ? Benjamin, Lyotard, Rancière, Paris, L'Harmattan, 2007, p. 46-47.

28. MediaArtHistories, Oliver Grau, ed., Cambridge, Mass.: MIT Press, 2007, p. 4.

29. Ctrl (space): Rhetorics of Surveillance from Bentham to Big Brother, Thomas Y. Levin, Ursula Frohne, Peter Weibel, eds., Karlsruhe: ZKM Center for Art and Media, Cambridge, Mass.: MIT Press, 2002. 
30. Giorgio Agamben, Qu'est-ce qu'un dispositif, Paris, Payot, Rivages, 2007, p. 42.

31. Gilles Deleuze, Félix Guattari, Mille plateaux. Capitalisme et schizophrénie 2, Paris, Éditions de Minuit, 1980, [2001], p. 434-527.

32. Gilles Deleuze, Claire Parnet, Dialogues, Paris, Flammarion, 1977, p. 39-49.

33. Site de InterActunet.net (Jan. 9, 2008).

34. Michel Maffesoli, « Une lecture de Georges Simmel », Sociétés, $n^{\circ} 74,2001 / 4$, p. 5-11.

35. La vie privée à l'heure des médias, Patrick Baudry, Claude Sorbets, André Vitalis, eds., Pessac, Presses universitaires de Bordeaux, 2002 ; Serge Tisseron, L'Intimité surexposée, Paris, Hachette, 2002 ; Id., Virtuel, mon amour: Penser, aimer, souffrir, à l'ère des nouvelles technologies, Paris: Albin Michel, 2008.

36. Merry Alpern, Dirty Windows, Zurich, Scalo, 1995 ; Nicholas Barker, Unmade beds, Stockport, UK : Dewi Lewis Pub., 1998 (publication sur le film : Unmade Beds, dirigé par Nicholas Barker, UK, 1997). 